\title{
A comparison of glucagon and glucose in prehospital hypoglycaemia
}

\author{
M A Howell, H R Guly
}

\begin{abstract}
Objective-To compare intramuscular glucagon with intravenous glucose in the prehospital management of hypoglycaemia in adults.

Methods-In the first part of the trial all UK ambulance services were asked how their personnel treat prehospital episodes of hypoglycaemia. In the second part, two protocols for treating prehospital hypoglycaemia were studied. In phase 1 , intramuscular glucagon $1 \mathrm{mg}$ was used. In phase 2 , intravenous glucose $25 \mathrm{~g}$ was used; if intravenous access was not possible, intramuscular glucagon was given.

Results-33 out of 43 respondent ambulance services $(76.7 \%)$ only use glucagon for prehospital hypoglycaemia; the remaining services use glucose and glucagon. In the second part of the study the median duration from diagnosis to full orientation (Glasgow coma score 15) was 28 minutes ( $95 \%$ confidence interval 18 to 49 minutes) in phase 1 and 11 minutes (95\% confidence interval 8 to 19 minutes) in phase 2. This difference is statistically significant $(P<0.005)$. On-scene times were not significantly different.

Conclusions-Intravenous glucose is the treatment of choice in prehospital hypoglycaemia but glucagon should also be available for intramuscular use when intravenous access is not possible. ( $f$ Accid Emerg Med 1997;14:30-32)
\end{abstract}

Keywords: glucagon; intravenous glucose; hypoglycaemia; prehospital treatment

Before this study, hypoglycaemia occurring within the Westcountry Ambulance Service region has been treated by National Health Service Training Division (NHSTD) trained paramedics with an intramuscular injection of glucagon $1 \mathrm{mg}$. In accordance with the local clinical management guidelines ${ }^{1}$ they then await a response. If the patient has not fully recovered within 10 minutes, has any injuries, or is alone, transfer to hospital is arranged. If the patient has fully recovered within 10 minutes, has a normal blood sugar, can take sweet drinks, and does not wish to attend hospital, they are advised to see their general practitioner or diabetic liaison nurse within 24 hours.

The time taken for hypoglycaemia to respond to glucagon has not been measured in the prehospital environment, but studies in hospital have shown that even when given intrave- nously, it is greater than following the administration of intravenous glucose. ${ }^{2}$

The first part of our study investigated how ambulance services in the United Kingdom treat prehospital hypoglycaemia and the second part aimed to compare the responses of hypoglycaemic patients treated with intravenous glucose with those treated with intramuscular glucagon.

\section{Methods}

Local Regional ethics committee approval was gained.

In the first part of the study the training officers of all ambulance services in the United Kingdom were sent a letter asking how their crews treat prehospital episodes of hypoglycaemia. In particular they were asked whether their vehicles carry glucagon, glucose, or both, in what forms, and if there are any plans to review their practices. A further letter was sent if no initial reply was forthcoming.

The second part of the study consisted of two consecutive three month periods. In the first phase, all patients over the age of 18 diagnosed as being hypoglycaemic in the catchment areas of Plymouth, Tavistock, and Kingsbridge ambulance stations were treated according to the existing protocol with intramuscular glucagon $1 \mathrm{mg}$ (Glucagon Novo). In the second phase, hypoglycaemia was treated with intravenous glucose $25 \mathrm{~g}$ in the form of 50 $\mathrm{ml}$ of a $50 \%$ solution (Glucose Min-I-jet). If intravenous access could not be achieved, glucagon was given intramuscularly.

Children were not included in the study because intravenous access is generally more difficult and NHSTD trained paramedics are not trained to perform paediatric cannulation.

Hypoglycaemia was defined as a blood sugar of less than $4 \mathrm{mmol} /$ litre, as measured by Glucostix.

Data were collected by use of a questionnaire attached to the drug packaging. Details of date, time, and patient demography were noted, along with patient symptoms, initial Glasgow coma scale score (GCS), and Glucostix reading. Timings of ambulance arrival, diagnosis, drug administration, recovery to full orientation (defined as GCS 15), and ambulance departure were collected. Patient disposal was also recorded.

\section{Results}

Of 51 ambulance services written to, 43 replied, a response rate of $84.3 \%$. Thirty three services $(76.7 \%)$ use glucagon only, while the remaining $10(23.3 \%)$ use glucagon and 


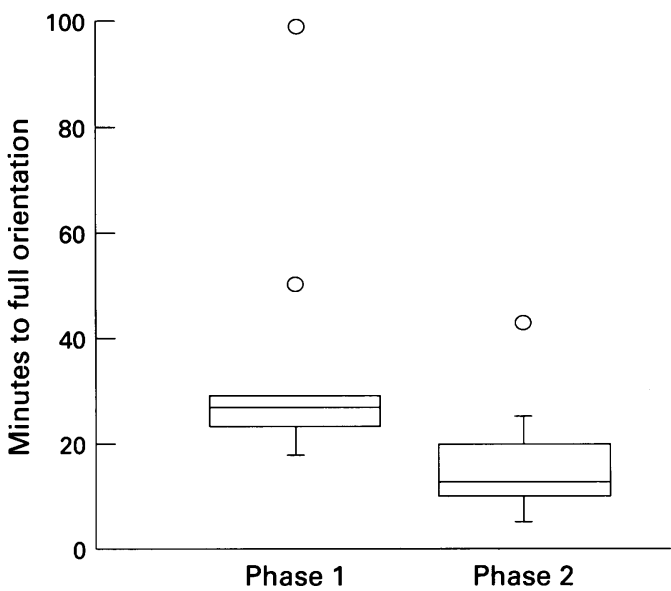

Times from diagnosis of hypoglycaemia to full orientation.

glucose. All but one of the latter services use 50 $\mathrm{ml}$ of glucose $50 \%$; the remaining service uses $250 \mathrm{ml}$ of glucose $10 \%$, an equivalent dose of glucose $(25 \mathrm{~g})$. Three ambulance services volunteered that they were currently reviewing their protocols for the management of hypoglycaemia.

In the second part of the study, nine patients were recruited in the first phase (February to April 1995) and 19 in the second phase (May to July 1995). Five of those in phase 2 required intramuscular glucagon due to failure to secure intravenous access. Four other hypoglycaemic patients were excluded from the trial; two who were satisfactorily treated with oral sugar, one victim of multiple trauma; and one with an associated haematemesis necessitating admission to the intensive care unit.

All patients were previously diagnosed insulin dependent diabetics and there were no statistically significant differences between the two groups in age, symptomatology, initial GCS, Glucostix reading, or time taken for diagnosis to be made.

The time from diagnosis to full orientation was studied. In phase 1 the median time was 28 minutes ( $95 \%$ confidence interval 18 to 49 minutes) and in phase 2 (including those requiring glucagon) it was 11 minutes $(95 \%$ confidence interval 8 to 19 minutes). These results are shown graphically in the figure. Statistical analysis using the extension of the median test ${ }^{3}$ yielded a $\chi^{2}$ value of 8.02 with one degree of freedom. This is statistically significant $(P<0.005)$.

In the 14 patients given glucagon in phase 1 and phase 2 the median time between giving glucagon and full recovery was 17.5 minutes ( $95 \%$ confidence interval 15 to 34 minutes). In phase 2 , for those given glucose, the median time to place an intravenous cannula was 3.5 minutes (95\% confidence interval 2 to $15 \mathrm{~min}$ utes) and the median time between giving glucose and full recovery was 3.0 minutes (95\% confidence interval 2 to 6 minutes).

The median on-scene time was 37 minutes in phase 1 and 36.5 minutes in phase 2 . This is not statistically different.

No patient in phase 1 was brought to hospital but three patients in phase 2 were brought to the accident and emergency department.

\section{Discussion}

From the results of our postal survey, in approximately three quarters of United Kingdom ambulance services, prehospital hypoglycaemia is treated by the administration of intramuscular glucagon $1 \mathrm{mg}$. If no response is obtained the patient is transferred to hospital for further treatment. The gold standard for the treatment of hypoglycaemia is intravenous glucose $25 \mathrm{~g}$ ( $50 \mathrm{ml}$ of a $50 \%$ solution). ${ }^{4}$ Glucagon, however, has several advantages in that it can be given intramuscularly by ambulance technicians and can be given to patients where intravenous access is difficult because of fitting, aggressive behaviour, or peripheral venous shutdown. Both glucose and glucagon have been studied in prehospital hypoglycaemia, ${ }^{5-7}$ but they have not been compared.

It is not unexpected that hypoglycaemia responded faster to intravenous glucose than to intramuscular glucagon, but when treating hypoglycaemia the important time interval is between diagnosis and regaining full consciousness. Siting an intravenous line takes a median of 3.5 minutes, which builds a delay into the response time of patients treated with intravenous glucose. This figure of 3.5 minutes to site an intravenous cannula is comparable with those of other studies showing that it takes between two and four minutes to site a cannula,${ }^{89}$ but the procedure adds between 12 and 14 minutes to on-scene time. ${ }^{10-13}$ However, even with this delay and including those patients in whom cannulation was not possible and therefore glucagon was required, the time between diagnosis of hypoglycaemia and regaining of full consciousness in phase 2 was significantly shorter than in phase 1 .

On-scene times in both phases of the study were longer than expected, but are similar to the mean of 32 minutes found by Cooke in a study of prehospital hypoglycaemia in the West Midlands. ${ }^{6}$ In accordance with local clinical management guidelines for the management of hypoglycaemia, ${ }^{1}$ ambulance paramedics await a response to treatment before making a decision to transport a patient to hospital.

This study, albeit small, shows the superiority of intravenous glucose, which should be regarded as the treatment of first choice for prehospital hypoglycaemia, but glucagon needs to be available to treat those patients in whom intravenous access cannot be secured.

Following extensive consultation with the local ambulance service trust, protocols are to be revised to take the findings of the study into account and $50 \%$ glucose will be carried by all front line crews.

We would like to thank the paramedics of Westcountry Ambulance Service Trust for their cooperation and Dr Xiao Ming Huang, Department of Information, Derriford Hospital, for statistical advice.

1 Westcountry Ambulance Services NHS Trust Clinical Management Guidelines.

2 Collier A, Steedman DJ, Patrick AW, Nimmo GR, Matthews DM, Macintyre CCA, et al. Comparison of intravenous DM, Macintyre CCA, et al Compar glucagon and dextrose in treatment of severe hypoglycaemia in an accident a

3 Siegel S, Castellan NJ. Nonparametric statistics for the behavioural sciences, 2nd ed. New York: McGraw-Hill, 1988:200-6. 
4 Weatherall DJ, Ledingham JGG, Warrell DA. Oxford textbook of medicine, 3rd ed, vol 2. Oxford: Oxford University Press, 1996:1498.

5 Weston C, Stephens M. Hypoglycaemic attacks treated by ambulance personnel with extended training. BMJ 1990; 300:908-9.

6 Yaxley L, Aldridge V, Almond J, Harradence F, Henry P, Heyburn PJ, et al. The treatment of severe hypoglycaemia with glucagon administered by ambulance personnel. Diawith glucagon administered by amt
betic Med 1991;8(suppl 1):71.

7 Cooke MW. Is intravenous dextrose appropriate in prehospital care? J Br Assoc Immed Care 1993;16:3-4.

8 O'Gorman M, Trabulsy P, Pilcher DB. Zero-time prehospital IV. J Trauma 1989;229:84-6.
9 Jones SE, Nesper TP, Alcouloumre E. Prehospital intravenous line placement: a prospective study. Ann Emerg Med 1989;18:244-6.

10 Johnson GS, Guly HR. The effect of pre-hospital administration of intravenous nalbuphine on on-scene times. J Accid Emerg Med 1995;12:20-22.

11 Donovan PJ, Cline DM, Whitley TW, Foster C, Outlaw M. Prehospital care by EMTs and EMT-Is in a rural setting: prolongation of scene times by ALS procedures. Ann prolongation of scene times

12 McSwain GR, Garrison WB, Artz CP. Evaluation of resuscitation from cardiopulmonary arrest by paramedics. Ann Emerg Med 1980;9:341-5.

13 Rouse A. Do ambulance crews triage patients? Arch Emerg Med 1991;1:185-91.

\section{EMERGENCY CASEBOOK}

Isolated traumatic radial head injury in children: a missed injury

Isolated traumatic dislocation of the radial head in children is an uncommon injury. The mechanism of injury involves hyperpronation of the forearm and a varus strain. The radial head commonly dislocates in an anterior or antelateral direction. The level of trauma involved is not necessarily severe and elbow flexion and extension may not be significantly reduced. It is, therefore, easy to miss this injury. The key to diagnosing the injury is a high degree of suspicion and proper interpretation of the elbow radiographs. Dislocation of the radial head can be identified by noting that the line traced from the radial shaft and head does not cross the centre of the capitellum (see the figures).
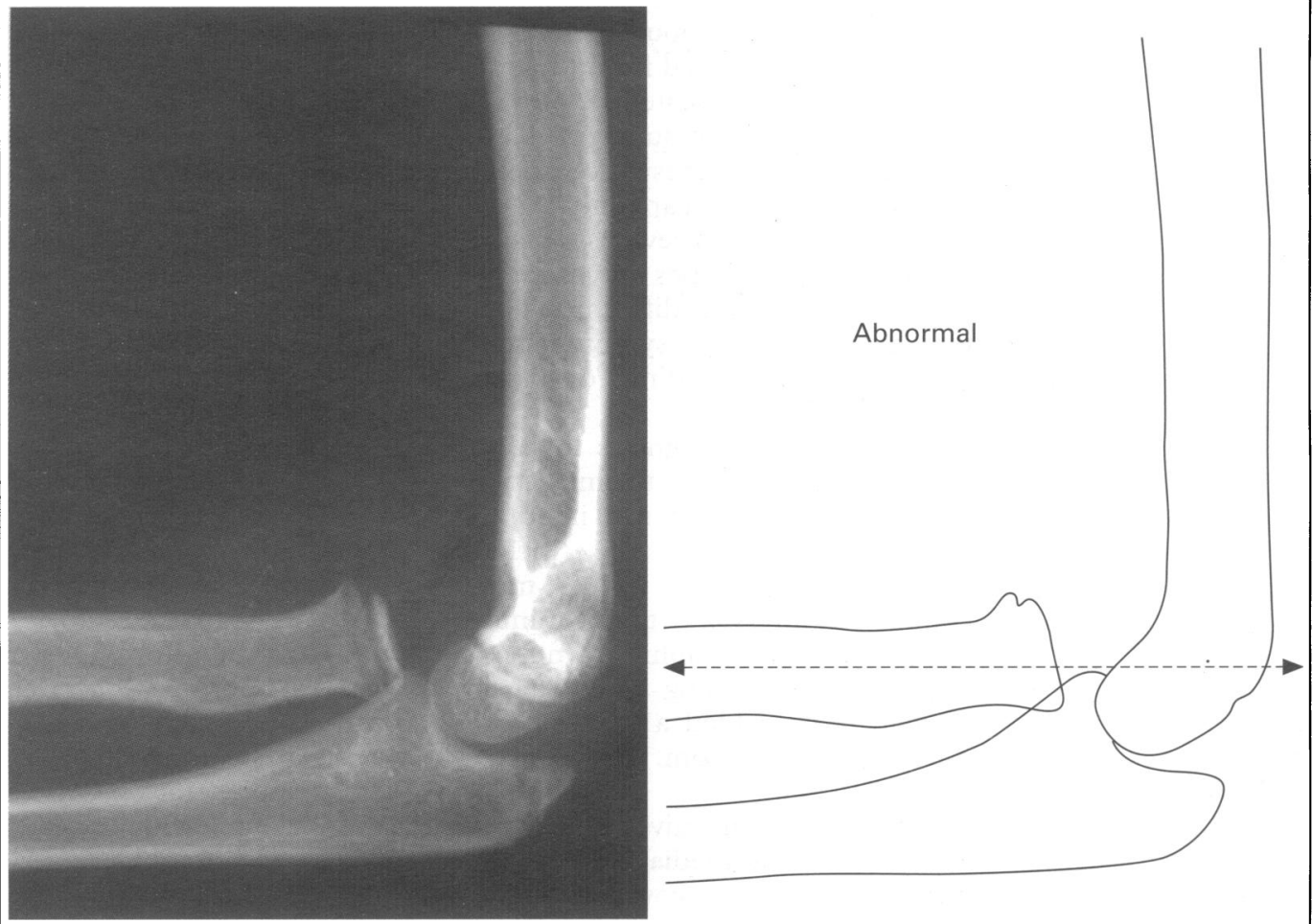

Once the diagnosis is made it is mandatory to obtain radiographs of the forearm to exclude a Monteggia injury or ulnar bowing. Closed reduction is usually easy to achieve and long term results are excellent. M SAAB, N M G KURDY, R BIRKINSHAw, Stockport Infirmary Hospital, Stockport. Correspondence: Mr M Saab FRCS, senior registrar, Accident and Emergency Department, North Manchester General Hospital, Delauney's Road, Crumpsall, Manchester M8 6RB. 